\title{
How aesthetically objectionable is fluorosis?
}

\author{
Aesthetically objectionable fluorosis in the United Kingdom \\ M. N. Alkhatib, R. Holt and R. Bedi Br Dent J 2004; 197: 325-328
}

\section{Objectives}

A cross-sectional national survey to explore perceptions of dental fluorosis and to determine the proportion of people regarding fluorosis as aesthetically objectionable at differing levels of defect.

\section{Methods}

A survey using a multistage stratified random probability sample of 6,000 UK adult households. Face-to-face interviews were carried out using a structured questionnaire and photographs of different levels of dental fluorosis. Respondents were interviewed about the parameters of satisfaction, attractiveness and need for treatment for dental fluorosis.

\section{Results}

The proportion of respondents perceiving teeth as unattractive, unsatisfactory and requiring treatment increased with increasing severity of dental fluorosis. Using agreement between the three negative perceptions as a measure, $14 \%$ of the sample perceived mild dental fluorosis as aesthetically objectionable, $45 \%$ at moderate level and 91\% at severe levels.

\section{Conclusion}

Negative perceptions of dental fluorosis were lower than reported previously. Three parameters were included in the approach to estimate aesthetically objectionable fluorosis which may provide a more realistic measure than those used previously. The nature of the index and the sample included suggest that findings of this survey provide a reasonable indicator of the likely impact of water fluoridation. Findings may have important implications for fluoridation policies in the United Kingdom and elsewhere.

\section{IN BRIEF}

This paper:

- Improves the understanding of the disadvantages of water fluoridation.

- Demonstrates public perceptions of dental discolouration typical of dental fluorosis.

- Shows in one step the application of public participation and involvement in health service research by gathering the public's views on a health issue.

- Provides findings that will assist policy makers to settle the issue of water fluoridation in the United Kingdom.

\section{COMMENT}

Increasingly patients and public opinions are being sought when assessing outcomes from dental treatment and public healthcare initiatives. ${ }^{1}$ Patients and the publics' viewpoints cannot and should not be assumed, and are important to consider in embracing patient partnership in oral health. Alkhatib et al.'s study is an interesting one in which they explore current perceptions of dental fluorosis in the United Kingdom. The study employs the Office for National Statistics (ONS) Omnibus Surveys to enable a large random sample of UK adults' perceptions on dental fluorosis to be considered, and this is a rapid and easy way to collect such information. The combination of three perceptions:

'attractiveness', 'satisfaction' and 'treatment need' provides a more comprehensive measure of acceptability and unacceptability of dental fluorosis compared with many other studies. As expected, perceived attractiveness, satisfaction, and need for dental treatment was strongly related to the degree of fluorosis which previous research has also reported.2,3 However, the current study provides a valuable 'snap shot' of opinion on dental fluorosis, providing baseline data for the UK on such matters. It is apparent that approximately a third of the UK public perceives even mild fluorosis to be unattractive (34\%), would be dissatisfied to have teeth with such appearance (36\%) and feel that mild fluorosis requires treatment (29\%). Clearly this has implication for fluoridation policies. Given the many types of variables collected (notably socio-demographic factors) in the ONS omnibus surveys it would have been interesting to determine socio-demographic variations in perceptions of dental fluorosis. Now that baseline data on aesthetic objectionable in the United Kingdom has been provided by this study it would be valuable to monitor changes in perceptions in relation to dental fluorosis over time and also to consider cross cultural differences in opinions of dental fluorosis.

C. McGrath, Associate Professor, Faculty of Dentistry, University of Hong Kong. doi:10.1038/sj.bdj.4811646

1. Buck D, Newton J T. Non-clinical outcome measures in dentistry: publishing trends 1988-98. Community Dent Oral Epidemio/2001;29:2-8.

2. Riordan PJ. Perceptions of dental fluorosis. J Dent Res 1993: 72: 1268-1274.

3. Ellwood R P, O'Mullane D. Enamel opacities and dental esthetics. J Public Health Dent 1995; $55: 171-176$. 